\title{
Niveles intelectuales y ansiedad en niños con intoxicación plúmbica crónica. Colegio "María Reiche". Callao-Perú, 2002
}

\author{
Julián Vega ${ }^{1}$, Juan De Coll ${ }^{1}$, Jorge Lermo ${ }^{1}$, Juan Escobar ${ }^{1}$, \\ Miriam Díaz ${ }^{1}$, José Castro ${ }^{2}$
}

\begin{abstract}
Resumen Objetivo: Determinar la asociación entre intoxicación plúmbica crónica y niveles intelectuales, y la asociación entre intoxicación plúmbica crónica y ansiedad, en niños del colegio "María Reiche”-Callao. Materiales y Métodos: Se realizó un estudio observacional transversal en 39 niños que participaron en el dosaje de plomo en sangre hecho por la Dirección General de Salud Ambiental en el año 2002. Se midió el coeficiente intelectual y determinó el nivel intelectual, y se aplicó la lista de chequeo de ansiedad. Se categorizó a los niños, según sus niveles de plomo en sangre, en no intoxicados (niveles $<10 \mu \mathrm{g} / \mathrm{dL}$ ) e intoxicados (niveles $\geq 10 \mu \mathrm{g} / \mathrm{dL}$ ). Se aplicó las pruebas chi-cuadrado y prueba t con un $p<0,05$. Resultados: El coeficiente intelectual de los niños intoxicados fue 85,51 $\pm 12,56$, los niveles de plomo fueron $26,54 \pm 9.42 \mu \mathrm{g}$ / $d L$ en los niños con nivel intelectual bajo (fronterizo e inferior). Los niveles intelectuales $y$ las categorías de plomo en sangre se asociaron con un $p=0,355$. Los grados de ansiedad y las categorías de plomo en sangre se asociaron con un $p=0,03$. La distribución t encontró diferencia de medias entre los niveles de plomo en los grupos con ansiedad leve y marcada $(p=0,024)$. Conclusiones: Se encontró asociación entre la intoxicación plúmbica crónica y el grado de ansiedad; sin embargo, no se encontró asociación entre los niveles intelectuales y la intoxicación plúmbica crónica.
\end{abstract}

Palabrasclave Envenenamiento por plomo; exposición a riesgos ambientales; enfermedades ambientales; ansiedad.

Intelectual levels and anxiety in children with chronic lead intoxication. Maria Reiche School. Callao-Peru, 2002

\section{Abstract}

Objectives: To determine the association between both chronic lead intoxication and intellectual levels and between chronic lead intoxication and anxiety in children from Maria Reiche School - Callao. Materials and Methods: A cross sectional and observational study was done in 39 children who had participated in a blood lead screening done by the General Direction of Environmental Health in 2002. The intellectual coefficient was measured

\footnotetext{
1 Alumnos de la Escuela Académico Profesional de Medicina Humana. Facultad de Medicina, UNMSM. Lima, Perú.

2 Docente del Departamento Académico de Medicina Preventiva y Salud
} Pública. Facultad de Medicina, UNMSM. Lima, Perú. and the intellectual level determined; the anxiety checkup list was applied. Children groups were classified according to their blood lead levels in non-poisoned (levels $<10 \mu \mathrm{g}$ ) $d L$ ) and poisoned (levels $\geq 10 \mu \mathrm{g} / \mathrm{dL}$ ). Chi square and $t$ Student tests and odds ratio were applied with $p<0,05$. Results: Poisoned children average intellectual coefficient was 85,5 $\pm 12,6$, and average blood lead levels in children with low intellectual coefficient (border and inferior) 24,4 10,9. The intellectual levels and the blood lead categories were related with $p=0,03$. Poisoned children presented high risk of deep anxiety $(O R=6,33$ [IC 95\% 1,001-40,071]). T distribution found differences between blood lead levels in the light and deep anxiety groups $(p=0,024)$. Conclusions: We found association between chronic lead intoxication and anxiety degree, but not between intellectual levels and chronic lead intoxication. Keywords: Lead poisoning; environmental exposure; environmental illness; anxiety. 


\section{INTRODUCCIÓN}

El plomo es un metal pesado que, aparentemente, no cumple papel alguno en la fisiología humana $\left({ }^{1}\right)$. Su mecanismo de toxicidad se basa en reemplazar al $\mathrm{Ca}^{2+}$ en el transporte iónico $\left({ }^{2}\right)$. Los adultos absorben entre 5 y $10 \%$ del plomo ingerido y sólo retienen el $5 \%$, mientras que los niños, debido a su rápido metabolismo, absorben el $40 \%$ y retienen el $30 \%\left({ }^{3}\right)$. La acumulación de plomo en el organismo se da principalmente en el hueso, por lo que es considerado una fuente de exposición endógena. En adultos, esta acumulación es cercana al $95 \%$, mientras que en niños la cifra es próxima a $70 \%\left({ }^{3,4}\right)$. La vida media del plomo en tejidos, como el riñón e hígado, oscila entre 20 y 30 días, mientras que en el cerebro es de aproximadamente 2 años, en los glóbulos rojos de 35 días y en el hueso varía entre 5 y 30 años $\left({ }^{1,4,5}\right)$. Los niveles de plomo en sangre que se admite como aceptables han sido reformulados a medida que se ha ampliado el conocimiento de los trastornos que produce este metal en el hombre. En la actualidad, el Centro de Control de Enfermedades de los Estados Unidos (CDC) aconseja que los habitantes en general deben tener niveles de plomo en sangre por debajo de los $10 \mu \mathrm{g} / \mathrm{dL}$, considerándose intoxicación por plomo a niveles mayores $\left(^{5}\right)$.

En la intoxicación plúmbica crónica (IPC), la población infantil es la más afectada; debido a que sus tejidos se encuentran en pleno desarrollo $\left({ }^{2,6}\right)$. El sistema nervioso es el principal tejido dañado, incluso a concentraciones bajas, encontrando los mayores niveles de plomo principalmente en la sustancia gris y los núcleos basales $\left({ }^{2}\right)$. La neurotoxicidad que produce la IPC conlleva a severos trastornos de las funciones cognitivas, que se expresan en problemas de aprendizaje y conducta y problemas neurológicos, como cefalea, disminución de la agudeza visual, alteraciones del lenguaje y retraso mental $(2,7)$.
El daño cognitivo asociado a la IPC se encuentra asociado a niveles de exposición presumiblemente seguros; es decir, en concentraciones en las cuales aparentemente no hay sintomatología. Los niveles de plomo en sangre inferiores a $10 \mu \mathrm{g} / \mathrm{dL}$ (rango menor al punto de corte de intoxicación plúmbica) están inversamente asociadas a los puntajes de coeficiente intelectual (CI) en niños de tres a cinco años $\left({ }^{5,8}\right)$. En algunos estudios, incluso, se menciona que existe una disminución de 1 a 3 puntos en el resultado obtenido de las pruebas de CI por cada $10 \mu \mathrm{g} / \mathrm{dL}$ que se incrementa en sangre. Esta alteración del CI persiste en la adultez cuando ha existido una exposición en edades tempranas de la vida. Otros problemas neuropsicológicos asociados a IPC son el déficit de atención, alteraciones del razonamiento abstracto, de la memoria verbal, de la fluidez verbal; la presencia de perseverancia (repetición de respuestas incorrectas), de conductas antisociales y delincuenciales y el daño de los sistemas cerebrales de regulación emocional y afectiva $\left({ }^{5}\right)$.

La Dirección General de Salud Ambiental (Digesa) encontró en el Callao que los niños de los colegios "Guadalupe" y "María Reiche" (ubicados cerca de una extensa área de almacenamiento de minerales) registraron una media de plomo en sangre de $40,7 \mu \mathrm{g} / \mathrm{dL}\left({ }^{9,10}\right)$. Observaron que el vivir o estudiar cerca a los depósitos de plomo aumenta en casi 18 veces el riesgo de tener valores elevados de plomo en sangre $\left({ }^{11}\right)$ y que existe una alta prevalencia de intoxicación por plomo $(93,4 \%)$ en los niños del AAHH "Puerto Nuevo", lugar donde se ubica el colegio "María Reiche". Es en los primeros 24 meses de vida cuando se observa la mayor velocidad de acumulación de plomo en el organismo $\left({ }^{12}\right)$.

En el presente estudio, se buscó determinar la asociación existente entre la intoxicación plúmbica crónica y los niveles intelectuales y la ansiedad en niños del Colegio "María Reiche", Callao-Perú. 


\section{MATERIALES Y MÉTODOS}

El tipo de estudio fue observacional y de corte transversal. El área de estudio fue el C.E. $\mathrm{N}^{\circ}$ 5045 María Reiche, centro de educación primaria ubicado en la Av. Contralmirante Mora $\mathrm{N}^{\circ}$ 420, AAHH "Puerto Nuevo", provincia constitucional-del-Callao.

La población de estudio estuvo conformada por la totalidad de los alumnos (241 niños) participando en el estudio 39 niños que se encontraban entre el $3^{\text {er }}$ y $6^{\circ}$ grado de educación primaria. Se tomó como criterios de inclusión pertenecer a la lista de dosaje de plomo en sangre realizado por Digesa en el año 2002, tener entre 8 y 12 años de edad y estar matriculado en el año académico 2002 de dicho colegio. El criterio de exclusión fue no haber aceptado participar en el estudio.

El método de cuantificación de plomo en sangre utilizado por Digesa fue el lead care (método rápido) y, a toda medición mayor de $30 \mu \mathrm{g} / \mathrm{dL}$, se le realizó la prueba de absorción atómica para determinar con exactitud el nivel de plomo correspondiente. Los niños fueron agrupados en dos categorías según sus niveles de plomo en sangre: no intoxicados (niveles $<10$ $\mu \mathrm{g} / \mathrm{dL}$ ) e intoxicados (niveles $\geq 10 \mu \mathrm{g} / \mathrm{dL}$ ) $\left(^{5}\right)$.

La medición del coeficiente intelectual (CI) y la determinación del nivel intelectual se realizó mediante la prueba Cattell factor "g" escala 2 para las edades de 8 a 14 años. Teniendo en cuenta los resultados encontrados por Altez $\left({ }^{13}\right)$, se reagrupó a los niños en tres categorías, de acuerdo al nivel intelectual en alto (muy superior, superior), medio (normal superior, normal y normal inferior) y bajo (fronterizo e inferior). Se aplicó también la lista de chequeo de ansiedad para niños de 0 a 12 años, recabada del Instituto de Investigaciones de la Facultad de Psicología de la Universidad Nacional Mayor de San Marcos y aprobado por el Centro Peruano de Investigaciones Psicológicas y Modificación del Comportamiento (CIPMOC). Esta lista evalúa el ámbito emotivo. Consistió en 26 ítems de alternativa múltiple, realizado en una entrevista estructurada, clasificándose en grados de ansiedad normal, ansiedad leve y ansiedad marcada.

Los resultados fueron analizados utilizando la prueba chi-cuadrado, la prueba t y correlación, para lo cual se utilizó el paquete estadístico SPSS versión 11,0 con un nivel de confianza de $95 \%$.

Para la realización del estudio se contó con la autorización de la Dirección del Centro Educativo No 5045 María Reiche y de los padres.

\section{RESULTADOS}

El total de niños evaluados fue 39 , siendo la media de edad 9,73 años; 14 fueron mujeres y 25 varones. La totalidad de los niños tuvo dosaje de plomo en sangre, estos niveles se encontraron entre 5 y $44,4 \mu \mathrm{g} / \mathrm{dL}$, con una media de 18,59 $\mu \mathrm{g} / \mathrm{dL}(\mathrm{DE} \pm 9,91)$.

Hubo diferencia de medias en las concentraciones de plomo en sangre con respecto al sexo mediante la prueba $\mathrm{t}(p=0,036)$.

Los valores de coeficiente intelectual se encontraron entre 60 y 112 , cuya media fue $87,79(\mathrm{DE} \pm 12,74)$. Ningún niño estuvo en el nivel intelectual alto (muy superior y superior); el $75 \%$ de los niños (27) se halló en el nivel intelectual medio (normal superior, normal y normal inferior).

La media de los valores del coeficiente intelectual de los niños no intoxicados fue de 92,89 (DE $\pm 12,3)$, asimismo, la media de los valores del coeficiente intelectual de los niños intoxicados fue 85,51 (DE $\pm 12,56)$.

$\mathrm{Al}$ correlacionar los concentraciones de plomo en sangre y los valores de coeficiente intelectual encontramos un $\mathrm{r}=-0,252(p=0,138)$.

Se encontró diferencia de medias entre las concentraciones de plomo en sangre de los niveles intelectuales medio y bajo $(p=0,022)$ mediante la prueba $\mathrm{T}$ (Tabla 1). 
Tabla 1. Nivel intelectual y promedio de niveles de plomo en sangre.

\begin{tabular}{lrcc}
\hline $\begin{array}{c}\text { Nivel } \\
\text { Intelectual }\end{array}$ & $\mathrm{n}$ & $\begin{array}{c}\text { Niveles de } \\
\text { plomo } \pm \mathrm{DE}\end{array}$ & $p$ \\
\hline Bajo & 9 & $\begin{array}{c}24,37 \pm 10,96 \\
16,16 \pm 7,97\end{array}$ & 0,022 \\
Medio & 27 & & \\
\hline
\end{tabular}

Al asociar los niveles intelectuales y las categorías según sus niveles de plomo en sangre (intoxicados y no intoxicados) mediante la prueba chi cuadrado se halló un $p=0,355$.

En la evaluación de ansiedad, hubo 22 niños (71\%) con grado de ansiedad marcada, de los cuales 19 presentaron intoxicación plúmbica, habiendo asociación entre las categorías de los niveles de plomo en sangre y los grados de ansiedad en la prueba chi cuadrado, con un $p=$ 0,03 (Figura 1). Así mismo, se halló diferencia de medias entre las concentraciones de plomo en sangre con respecto a los grados de ansiedad

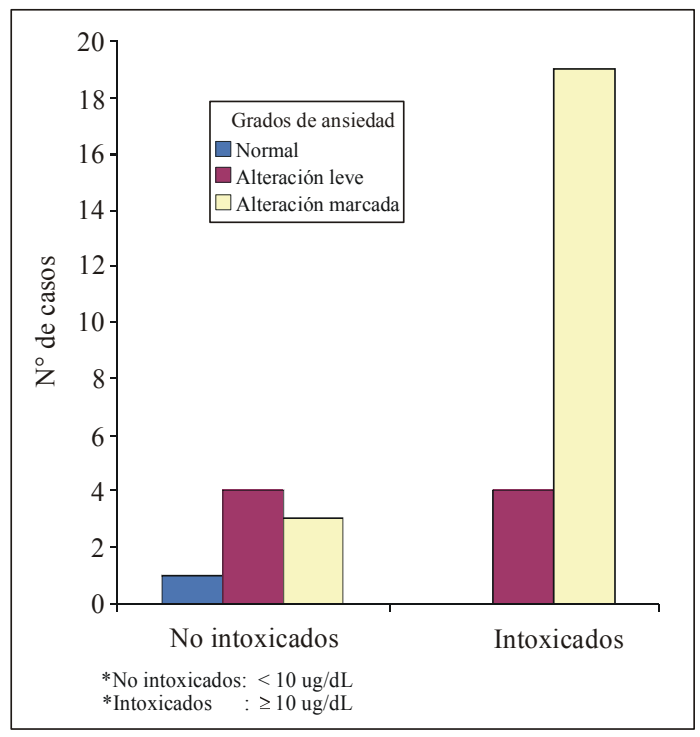

Figura 1. Distribución de grados de ansiedad y categorías según el nivel de plomo en sangre.
Tabla 2. Grados de ansiedad y promedio de niveles de lomo en sangre.

\begin{tabular}{lrcc}
\hline $\begin{array}{c}\text { Grados } \\
\text { de ansiedad }\end{array}$ & $\mathrm{n}$ & $\begin{array}{c}\text { Niveles de } \\
\text { plomo } \pm \mathrm{DS}\end{array}$ & $p$ \\
\hline Marcado & 22 & $20,20 \pm 9,77$ & 0,024 \\
Leve & 8 & $11,61 \pm 4,01$ & \\
Normal & 1 & 7,20 & \\
\hline
\end{tabular}

leve y marcado, mediante la prueba $\mathrm{t}$ con un $p$ $=0,024$. (Tabla 2).

\section{DISCUSIÓN}

Investigaciones epidemiológicas han revelado que la intoxicación plúmbica crónica se asocia al deterioro de las funciones cognitivoconductuales y a alteraciones del desarrollo en los niños $\left({ }^{14}\right)$. Este conocimiento ha servido en la interpretación de la necesidad de reducir o anular la exposición a este elemento $\left({ }^{12}\right)$.

Se encontró que el promedio de los niveles de plomo en sangre fue mayor en los niños con nivel intelectual bajo (fronterizo y deficiente); asimismo $88 \%$ (8) de estos niños estaba intoxicado. El $60 \%$ de los niños intoxicados presentó un coeficiente intelectual por debajo de lo normal, coincidiendo con el estudio de Pocock y col. en Inglaterra, que evidenció una disminución del coeficiente intelectual en niños con intoxicación plúmbica $\left({ }^{15}\right)$.

Los niños presentaron mayores niveles de plomo en sangre en relación a las niñas; esto coincide con otro estudio realizado en el AAHH "Puerto Nuevo", en el que se sostiene que las diferentes prácticas de juego y hábitos de interacción con el medio ambiente condicionarían diferentes grados de exposición al metal en ambos sexos $\left({ }^{16}\right)$.

Altez y col. $\left({ }^{17}\right)$ evaluaron a 847 niños en Lima, encontrando una mayor distribución de los niveles intelectuales normal superior, normal 
y normal inferior, que coincidió con nuestros resultados, donde $75 \%$ de los niños se ubicó en el mismo rango.

Nuestro estudio no halló una correlación inversa entre los niveles de plomo y el coeficiente intelectual, que discrepa con Canfield y col. que evaluaron a 172 niños y encontraron una relación inversa entre los niveles de plomo en sangre y el coeficiente intelectual, incluso en niños que presentaron niveles menores de 10 $\mu \mathrm{g} / \mathrm{dL}\left({ }^{8}\right)$.

Taboada $\left({ }^{18}\right)$ sostiene que la prevalencia de trastornos de ansiedad en la infancia es más alta en las niñas que en los niños, discordando con los resultados obtenidos en el presente estudio, donde 20 varones $(65 \%)$ presentaron ansiedad leve y marcada.

Un estudio en Lima evaluó a 112 individuos de 12 a 14 años y encontró síntomas que sugieren síndromes ansiosos entre 10 y $30 \%$, cifras menores a los resultados del presente estudio, en el que 3 de cada 4 niños presentaron ansiedad marcada $\left({ }^{19}\right)$.

Hubo relación entre los niveles de plomo y los grados de ansiedad, semejándose a los resultados obtenidos en el estudio de Needleman $\left({ }^{20}\right)$ en varones de una escuela pública en Pittsburg, entre las edades de 7 a 11 años, en el que se observó que con el pasar de los años, según aumentaba la cantidad acumulada de plomo aumentaban los reportes de agresividad, ansiedad y depresión.

Las limitantes del presente estudio fueron el no contar con el tamaño de muestra adecuado para poder inferir los resultados a la población estudiada y el no controlar factores que pueden disminuir el coeficiente intelectual en niños, tales como peso bajo al nacer, nivel intelectual de los padres, factores socioeconómicos, nivel nutricional, entre otros.

Como conclusión, los niños con niveles intelectuales bajos presentaron altos niveles de plomo en sangre; sin asociación entre intoxicación plúmbica crónica y los niveles intelectuales bajos. Se encontró asociación entre la intoxicación plúmbica crónica y los grados de ansiedad.

\section{AGRADECIMIENTOS}

Agradecemos al Lic. Milciades Ochoa Director del Colegio María Reiche y a los padres de familia por el apoyo y facilidades brindadas para el desarrollo del presente estudio.

\section{REFERENCIAS BIBLIOGÁFICAS}

1. Ignacio A. Intoxicación por plomo en pediatría. Arch Pediatr Urug. 2001;72(2):133-8.

2. Goodman A, Ruddon W, Molinoff B, Limbird E, Hardman G. Las bases farmacológicas de la terapéutica $9^{\circ} \mathrm{Ed}$. México, DF: McGraw-Hill Interamericana; 1998. p. 175561.

3. Dirección General de la Salud Ambiental. Estudio de plomo en sangre en una población seleccionada de la Oroya. Lima: DIGESA Perú; 1999. p. 5.

4. Sanín L, González-Cossío T, Romieu I, Hernández-Avila M. Acumulación de plomo en hueso y sus efectos en la salud. Salud Pública Mex. 1998;40:359-68.

5. Lidsky T, Schneider J. Led neurotoxicity in children: basic mechanisms and clinical correlates. Brain. 2003;126:5-19.

6. Rivas F, Fernández N. Exposición urbana no ocupacional al plomo y niveles sanguíneos en embarazadas y recién nacidos. [Tesis doctoral]. Mérido: Departamento de Farmacología y Toxicología, Facultad de Medicina, Universidad de los Andes; 1999.

7. Feldman RG. Effect of toxins and physical agents on the nervous system. En: Bradley WG, Daroff RB, Fenichel GM, Marsden CD (eds). Neurology in Clinical Practice. Boston: Butterworth-Heinemenn; 1991. p. 1185-209.

8. Canfield R, Henderson R, Cory-Slechta D, Cox Ch, Jusko $\mathrm{T}$, Lanphear B. Intellectual impairment in children whit blood lead concentrations below 10ìg per deciliter. N Engl J Med. 2003;348:1517-26.

9. Hernández M, Wegner S. Estudio demográfico de salud de niños intoxicados por plomo tercer dosaje AAHH «Puerto Nuevo»-Callao. Lima: Dirección General de Salud Ambiental; 2002.

10. Narciso J, Gaztañaga C, Espinoza R, Sánchez C, Moscoso S, Quequejana J, Hernández M. Estudio para determinar las fuentes de exposición de plomo en la provincia constitucional del Callao. Reporte final. Lima: DIGESA; 
junio 2000. Activity Report $N^{0} 104$. Financiada por the Bureau for Global Programs, Field Support and Research Office of Health and Nutrition U.S. Agency for International Development Washington, DC 20523.

11. Hernández M, Espinoza R, Moscoso S, Ortiz G, Lázaro B, Vigil L. Determinantes de los niveles de plomo en sangre en mujeres en el posparto temprano y niños de 6 meses a nueve años de edad en Lima y Callao. Reporte final. Lima: DIGESA; setiembre 1999. Activity Report. No 72. Financiada por the Bureau for Global Programs, Field Support and Research Office of Health and Nutrition U.S. Agency for International Development Washington, DC 20523.

12. López J. Intoxicación por plomo en niños menores de seis años en un asentamiento humano del Callao. An Fac Med Lima. 2000;61:37-45.

13. Altez I. Validación del test de inteligencia Cattell factor "g" escala 2. [Tesis de Licenciatura en Psicología]. Lima, Perú: Universidad Femenina Sagrado Corazón; 1995. p. 207.

14. Villeda-Hernández J. Efectos neurotóxicos en niños intoxicados con plomo. Arch Neurocien. 2002;7(2):90-8.

15. Pocock SJ, Smith M, Baghurst P. Environmental lead and children's intelligence: a systematic review of the epidemiological evidence. British Medical Journal. 1994;309:1189-97.
16. Dirección General de la Salud Ambiental. Estudio de plomo en sangre en una población seleccionada de Lima y Callao. Lima: DIGESA Perú; 1999. p. 3-11.

17. Altez I, Cano L, Pelaez D, Zambrano J. Estandarización de pruebas de aptitudes e inteligencia Cattell y Thorndike en niños. Lima: Unidad de Investigación. Facultad de Psicología, UNIFE; 1997. p. 113.

18. Taboada J, Ezpeleta A. Trastornos por ansiedad en la infancia y adolescencia: Factores de riesgo. Ansiedad y Estrés. 1998;4(1):1-16.

19. Perales A, Sogi C, Salas R. Salud mental en adolescentes del distrito de Magdalena. Monografías de investigación $\mathrm{N}^{\circ}$ 4. Lima: Instituto Nacional de Salud Mental Honorio Delgado-Hideyo Noguchi; 1996. p. 44-6.

20. Needleman HL, Riess JA, Tobin MJ, Biesecker GE, Greenhouse JB. Bone lead levels and delinquent behavior. JAMA. 1996;275(5):363-70.

Manuscrito recibido el 15 de abril de 2005 y aceptado para publicación el 30 junio de 2005.

Correspondencia: Dr. José Castro Quiroz

D. A. de Medicina Preventiva y Salud Pública.

Facultad de Medicina, UNMSM.

Av Grau 755. Lima 1, Perú.

Correo-e: jcastroq@minsa.gob.pe 